\title{
Extending the Chemical Vision in Life Science Using Water Cluster Ion Beam Secondary Ion Mass Spectrometry ( $\mathrm{H}_{2} \mathrm{O}-\mathrm{GCIB}$ SIMS)
}

Hua Tian

Pennsylvania State University, University Park, Pennsylvania, United States

Understanding the function of bio-systems (e.g., cells and tissues) and their interaction with exogenous compounds requires the ability to visualize spatial distribution of the biomolecules (e.g., lipids, small metabolites) and compounds at subcellular resolution. However, the routine laboratory assays are largely done by bulk analysis on extraction from dissociated cells and tissues, in which the spatial distribution is lost. We have been developing cluster ToF-SIMS for high resolution $(<1 \mu \mathrm{m})$ imaging of intact biomolecules (up to $\mathrm{m} / \mathrm{z} 5000$ ). Until recently, this has been brought to reality through our R\&D with high energy gas cluster ion beams (e.g. $70 \mathrm{keV}\left(\mathrm{H}_{2} \mathrm{O}\right){ }_{n}{ }^{+}(\mathrm{n}>28,000)$ ), the ionization source for mass spectrometry. Coupled with a DC beam buncher-ToF instrument ${ }^{1}$, the development bridges the gap between high spatial resolution and detection of intact larger biomolecules, while other contemporary imaging mass spectrometry could only satisfy one of those requirements.

With this setup, normal and diseased rat brain tissue (e.g., traumatic brain injury) are mapped at $1.5 \mu \mathrm{m}$ resolution for the first time to localize intact biomolecules and their peroxides up to $\mathrm{m} / \mathrm{z} 3000$ (e.g., gangliosides, cardiolipin (CL) and phosphatidylethanolamine (PE)). CL, a mitochondrial specific phospholipid, is indispensable for the integrity of the inner mitochondrial membrane and possesses vital roles in signaling. CL oxidation is a required stage of intrinsic apoptosis. PE peroxides generated in the endoplasmic reticulum (ER) are strong predictive biomarkers of ferroptosis. This is a step further for understanding the function of cardiolipins (m/z 1300 1500, CLs) and phosphoethanolamine (PE) peroxide in programmed cell death at a subcellular level ${ }^{2-3}$. The HeLa cells are imaged in a frozenhydrated state 3-dimentionally to elucidate small metabolites in de novo purine biosynthesis (DNPB) for the first time within single cells, revealing that DNPB carried by the multi-enzyme complex purinosomes that act as a metabolon to channel the pathway intermediates for the synthesis of purine nucleotides 4 . This recent development of cluster ToF-SIMS imaging opens new opportunities for multi-omics, cell heterogeneity and disease mark and target, leading to the further understanding of disease progress and development of new treatment.

\section{References}

1. Fletcher, J. S.; Rabbani, S.; Henderson, A.; Blenkinsopp, P.; Thompson, S. P.; Lockyer, N. P.; Vickerman, J. C., A New Dynamic in Mass Spectral Imaging of Single Biological Cells. Anal. Chem. 2008, 80 (23), 9058-9064.

2. Tian, H.; Sparvero, L. J.; Blenkinsopp, P.; Amoscato, A. A.; Watkins, S. C.; Bayir, H.; Kagan, V. E.; Winograd, N., Secondary-Ion Mass Spectrometry Images Cardiolipins and Phosphatidylethanolamines at the Subcellular Level. Angew. Chem. Int. Ed. Engl. 2019, 131, 3188-3193.

3. Tian, H.; Sparvero, L. J.; Amoscato, A. A.; Bloom, A.; Bayır, H.; Kagan, V. E.; Winograd, N., Gas Cluster Ion Beam Time-of-Flight Secondary Ion Mass Spectrometry High-Resolution Imaging of Cardiolipin Speciation in the Brain: Identification of Molecular Losses after Traumatic Injury. Anal. Chem. 2017, 89 (8), 4611-4619.

4. Pareek, V.; Tian, H.; Winograd, N.; Benkovic, S. J., Metabolomics and Mass Spectrometry Imaging Reveal Channeled De Novo Purine Synthesis by Purinosomes. Science 2020. 